\title{
Ontische $^{1}$ und pragmatische Fragen zur mittelalterlichen Textsorte Testament aus textlinguistischer Perspektive
}

\section{Joanna SMEREKA \\ Dr.; Universität Warschau; E-Mail: joannasmereka@op.pl}

\begin{abstract}
In my contribution it's about testaments made by german citizen of Cracow. The material is based on texts of last wills of people representing a minority of citizen of Cracow, the patrician families. The tastators where drawn dealer and trademans, which also pushing the development of the region. The texttype testament often marginalized by linguistics. I aske in opposite of this from the perspective of ontology and pragmatism: What is the deeper linguistic structure of this relative formalized texttype. I show in my analysis the texts of a last will include not only declarative (resp. indirect declarative) acts of speech but also a variety of assertive and directive elements. I don't want to decide the justice of an act of speech but I have a catalogue of repetitive facultative phrases and combine this with particular narrations of certain paragraphs of testaments. This element not only refers to the private sphere of the tastators (conflicts or emotions) but uncover the social, educational and in part religious attitude of the testators. And so at least fully developed testaments let understood as selfreports.
\end{abstract}

Keywords: testament, Cracow, middle ages, type of text, historical text-linguistics, pragmatism, selfreport

${ }^{1}$ Die ontische (daseinsmäßige) Perspektive bezieht sich im Unterschied zur ontologischen (seinsmäßigen) auf die Art und Weise, wie Menschen ihr Dasein an einem bestimmten Ort und zu einer bestimmten Zeit gestalten. Vgl. Heidegger, Martin: Sein und Zeit. Tübingen 1927. 


\section{Das Forschungsmaterial}

Die Grundlage meiner Forschung bildet ein einmaliges, historisches Forschungsmaterial, nämlich Krakauer Testamente deutschsprachiger Bürger, die in der Zeit zwischen den 90erJahren des 14. Jahrhunderts bis Mitte des 16. Jahrhunderts in die Krakauer Stadtbücher eingetragen wurden. Die in Krakau sesshaft gewordenen Deutschen waren willkommene Gäste, die - anfangs durch wirtschaftliche und stadtrechtliche Privilegien angelockt - als gelernte Handwerker und Kaufleute auch zur Entwicklung und zum Wohlstand der Stadt beitrugen. Die genaue Anzahl der Ankömmlinge ist heute nicht mehr vollständig rekonstruierbar. Schätzungsweise waren jedoch drei Viertel der zugezogenen Bürger im 14. und am Anfang des 15. Jahrhunderts deutscher Abstammung. ${ }^{2}$ Gegen Ende des 14. Jahrhunderts machten deutschsprachige Testamente daher auch 40 Prozent aller in die Krakauer Stadtbücher eingetragenen Texte dieser Textsorte aus. Insgesamt konnte ich bis 1550 eine Anzahl von 342 Testamenten ermitteln, die von mir gesichtet, transliteriert und tiefensprachlich analysiert wurden ${ }^{3}$. Obwohl das letzte auf Deutsch verfasste Testament das Eröffnungsdatum 1644 trägt, kann man anhand des erhobenen Quellenmaterials die Mitte des 16. Jahrhunderts als das Ende des Gebrauchs des Deutschen als Amtssprache in der Krakauer Stadtkanzlei annehmen, da ab diesem Zeitpunkt keine protokollartigen Eintragungen auf Deutsch in den Stadtbüchern zu finden sind. Die Spezifik der Materiallage in Krakau bedingt, dass für die Analyse die Texttypen Eintrag und Abschrift einer Urkunde ${ }^{4}$ ausgewertet werden konnten.

${ }^{2}$ Vgl. Kaczmarczyk, Kazimierz (Hg.): Księgi przyjęć do prawa miejskiego 1392-1506. Kraków 1913, S. 22.

3 Vgl. meine Dissertation Textlinguistische Untersuchungen zu Testamenten der Krakauer Bürger vom 14. bis zur Hälfte des 16. Jahrhunderts (i. Dr.). Der vorliegende Beitrag ist eine Fortführung der durchgeführten Untersuchung.

4 Die Terminologie und Unterscheidung übernehme ich von Spáčilová, Libuse: Deutsche Testamente von Olmützer Bürgern. Wien 2000. 


\section{Testamente als Verschriftlichung einer sozialen Handlung}

Bei der Beschreibung einer Textsorte ist man üblicherweise von ihrer Struktur und sprachlichen Ausgestaltung der sich in einzelnen Texten wiederholenden Sinnelemente ausgegangen. Eine solche Vorgehensweise wird auch angewendet, wenn historische Textmuster untersucht werden. Das Hauptgewicht legt dabei der Germanist Jörg Meier, der gemeinsam mit Arne Ziegler den Internationalen Arbeitskreis Kanzleisprachenforschung leitet, auf die äußere Gestaltung des Textes, da ,der Text im Kommunikationsprozess von den Teilnehmern zunächst von außen erfahren und zugeordnet wird ${ }^{\text {“5 }}$ und die Textarchitektur besonders die Makrostruktur als Globalstruktur einen identifizierenden Charakter aufweist. Das Textverstehen ist wiederum mit dem Erkennen und Verstehen einzelner Sinnelemente (kommunikativer Ganzheiten) verbunden, weshalb auch dieser Mikrostruktur eine wesentliche Bedeutung zukommt. Für die Eruierung dieser Elemente kann bei mittelalterlichen Kanzleitexten und sog. Bürgerurkunden, zu denen auch Testamente gehören, von der klassischen Diplomatik mit ihrem Urkundenschema ausgegangen werden. Diemikroskopischen Sinnelemente werden dabei unter stilistisch-lexikalischem Aspekt betrachtet, um die Formulierungsmuster, die sich in konventionellen Textsorten etabliert haben, zu ermitteln. Barbara Sandig betonte bereits in den 1970-er Jahren den inhärenten Zusammenhang zwischen der Realisierung einer sprachlichen Handlung und der Wahl einer bestimmten Art von Äußerung. ${ }^{6}$ Von Bedeutung ist dabei die Feststellung, dass die Befolgung eines

5 Meier, Jörg: Städtische Kommunikation in der Frühen Neuzeit. Historische Soziopragmatik und Historische Textlinguistik. Frankfurt am Main 2004, S. 53.

6 Sandig, Barbara: Stilistik. Sprachpragmatische Grundlagen der Stilbeschreibung. Berlin 1978, S. 16. 
Formulierungsmusters zur Realisierung eines bestimmten Textmusters führt. ${ }^{7}$

Bei relevanten, oft schriftlich fixierten sozialen Handlungen spricht der Warschauer Sprachforscher Józef Wiktorowicz von Handlungsmustern als Wiederholung bestimmter lexikalischsyntaktischer Konstruktionen. ${ }^{8}$ Die von Heinemann/Viehweger (1991: 17) postulierte Öffnung der Linguistik bedingt, dass sich „sich die Textlinguistik auf die Erforschung von Textstrukturen und Formulierungen beschränken [muss], jeweils in ihrer Einbettung in kommunikative, soziologische und psychologische Zusammenhänge“99.

Etwas deutlicher drückt diesen Gedanken Wolf-Dieter Krause (2010) aus, nach dem nicht nur die Entwicklung und Veränderung der Architektonik, also die äußere Gestaltungsweise, und die Komposition, also die Strukturierung der Inhaltsseite, sondern auch der Zustand der Textwelt von wissenschaftlichem Interesse ist. Die äußeren und inneren Strukturen bedingen sich gegenseitig. Die Textwelt ist ,im starken Maße von den politischen, ideologischen, religiösen und moralischen Bedingungen einer Gesellschaft" (S. 45) abhängig ${ }^{10}$.

Die polnische Sprachwissenschaftlerin Maria Wojtak schlägt eine Textsortenbeschreibung auf vier Ebenen vor, die sich in der Praxis oft überlappen: die strukturelle, die pragmatische, die stilistische und die ontologische (ontologisch-axiologische)

${ }^{7}$ Ebd., S. 20.

${ }^{8}$ Vgl. Wiktorowicz, Józef: Kanzleistil als Eigenschaft sprachlicher Handlungsmuster. Sprachliche Handlungsmuster in kanzleisprachlichen Texten. In: Hünecke, Rainer/Sandra Aehnelt (Hgg.): Kanzlei und Sprachkultur. Wien 2016, S. 97-106.

9 Zitiert nach Ziegler, Arne: Städtische Kommunikationspraxis im Spätmittelalter. Historische Soziopragmatik und Historische Textlinguistik. Berlin 2003, S. 322.

${ }^{10}$ Krause, Wolf-Dieter: Text, Textsorte, Textvergleich. In: Adamzik, Kirsten: Textsorten. Reflexionen und Analysen. Tübingen 2010, S. 45-76. 
Beschreibungsebene. ${ }^{11}$ An erster Stelle nennt die Lubliner Textlinguistin die von ihr wohl als wichtigste (am meisten konventionalisierte) erachtete Eigenschaft der Texte, die sie im Textaufbau der ältesten amtlichen polnischen Texte belegt hat. ${ }^{12}$ Was die formalisierten Texte anbelangt, zu denen die Testamente des späten Mittelalters und der frühen Neuzeit gehören, so kann man von einer Tendenz zur Vereinheitlichung einzelner Textexemplare ausgehen, da ihre Emittenten (nicht zu verwechseln mit Auftraggebern) professionelle Schreiber waren. Damit erweist sich die polnische (polonistische) Textologie bei der Frage nach dem Verhältnis von sprachlicher Handlung und ihrer Verschriftlichung als wegweisend, da durch die Einbettung in die sozialen Interaktionen Texte als Teil des Diskurses charakterisiert werden. ${ }^{13}$ Das Verständnis eines Textes verschiebt sich damit von einem stark distinguierenden Unterfangen in eine Richtung, die Texte als kommunikatives Ereignis begreift. ${ }^{14}$ Es kommt folglich zu einer Verflechtung von Kommunikationsakt, insbesondere der Sprechhandlung, und dem Produkt derselben, also dem Text.

Zwar verfolgt auch der Warschauer Germanist Józef Wiktorowicz einen ähnlichen Ansatz, allerdings mit dem Unterschied, dass er sich auf deutsche Autoren bezieht, ohne jedoch den Akteuren der Verschriftlichung die Aufmerksamkeit zuteil werden zu lassen, die sie verdienen. Er definiert die Textsorte Testament nämlich als eine Verschriftlichung der Rechtshand-

${ }^{11}$ Vgl.u.a. Wojtak, Maria: Genologia testów użytkowych. In: Ostaszewska, Danuta/Romuald Cudak (Hgg.): Polska genologia lingwistyczna. Warszawa 2008, S. 339-352; Wojtak, Maria: Gatunki prasowe. Lublin 2004, S. 16-17.

${ }^{12} \mathrm{Vgl}$. Wojtak, Maria: O stylowym wariancie języka na przykładzie tekstów urzędowych. In: Gajda, Stanislaw (Hg.): Systematyzacja pojęć w stylistyce. Opole 1992, S. 141-147, hier S. 145.

${ }^{13}$ Ebd., S. 78.

${ }^{14}$ Ostaszewska, Danuta/Cudak, Romuald (Hgg.): Polska genologia lingwistyczna. Warszawa 2008, S. 17. 
lung des Testierens, die eine rechtlich geregelte Situationskonstellation impliziert ${ }^{15}$. Ob sich diese Rechtshandlung in der Vertextung realisiert, oder ob die Vertextung sekundär zu dokumentarischen Zwecken von den Vertretern städtischer Kanzleien in einem Aktenbuch vorgenommen wird, ist für ihn bei der Textsortenklassifikation ohne Belang, da als Klassifikationsmaßstab die vorausgehende soziale Situation gilt und nicht deren Verschriftlichung. Für meine Betrachtung spielt jedoch die ontologisch-axiologische Ebene, die Maria Wojtak (2008) berücksichtigt, eine bedeutende Rolle bei der Untersuchung der Krakauer Testamente. Darunter fasse ich alle familiären, religiösen und existenziellen Aspekte zusammen, die in den Testamenten auszumachen sind. Es handelt sich folglich um das Weltbild, das im Text dargestellt wird, um das Wertesystem und um kulturgeschichtliche Faktoren. Eine Analyse aus der Sicht dieser Perspektiven ist zwar in dem Ausmaß, wie es Anna DuninDudkowska (2014) am Beispiel des Vergleichs gegenwärtiger amerikanisch-polnischer Texte vorführt, kaum möglich, da sowohl die Korpusgrundlage wie auch deren zeitliche Einbettung, eine (genaue) Rekonstruktion der in den ausgewerteten Textsorten vorkommenden Welt- und Wertvorstellungen, die über Jahrhunderte zurückliegen, nicht zulassen. Das Prinzip der Untersuchung kann jedoch übernommen werden: Erfasst und mit Beispielen belegt werden fakultative Elemente der Testamente und zwar unter Berücksichtigung einer möglichen kulturell-/mentalitätsgeprägten Begründung ihres Aufkommens. ${ }^{16}$ Deswegen soll diese vierte Beschreibungsebene der historischen

${ }^{15}$ Vgl. Wiktorowicz, Józef: Die Textsorte Testament in der deutschen Kanzleisprache in Krakau. In: Simmler, Franz (Hg.): Textsortentypologien und Textallianzen von der Mitte des 15. bis zur Mitte des 16. Jahrhunderts. Berlin 2004, S. 167-176.

${ }^{16}$ Vgl. Dunin-Dudkowska, Anna: Testament jako zwierciadło kultur. Lublin 2014. 
Textsorte Testament weiterhin Gegenstand (sozial-)geschichtlicher Untersuchungen sein. ${ }^{17}$

\section{Aufbau der testamentarischen Eintragungen in den Krakauer Stadtbüchern}

Die Eintragungen der Testamente in die Krakauer Stadtbücher erfolgten in die Acta Scabinalia (die von dem Gericht geführt wurden, gekürzt AS), in die Acta Consularia (die Bücher des Stadtrates, AC) und in die Liber Testamentorum (ebenfalls Bücher des Rates, LT 772 und LT 773). In den Krakauer Archivbeständen sind drei Arten von testamentarischen Eintragungen zu unterscheiden: a) Protokolle von mündlichen Testamentsablegungen vor der Behörde bzw. deren Vertretern (insgesamt 191 Stück: 83 in den AS, 88 bzw. 89, wenn ein gemischter Texttypus mitgerechnet wird, in dem LT 772 und 19 in den AC), b) Protokolle mit einer abgeschriebenen und in den Protokolltext inkorporierten Testamentsskizze, die der Behörde von außerhalb eingebracht wurde (sog. Czedel, insgesamt 76 Stück: 58 in den AS, 13 in dem LT 772 und 6 in den AC), und c) Abschriften der privaten Testamentsurkunden, die entweder im Amt eingelegt (c1verschlossene Testamente) oder diesem nur vorgelegt (c2 offene Testamente) wurden (insgesamt 50 Stück: 33 in den AS, 10 in dem LT 772 und 7 in dem LT 773). Mit einzurechnen sind 25 Testamente, die in Form einer Urkunde der jeweiligen Urkunde mit dem Ziel, noch größerer Sicherheit zu erreichen, in eines der Stadtbücher abgeschrieben wurden (3 in den AS, 8 in dem LT 772 und 14 in den LT 773). Im Untersuchungskorpus kommen insgesamt 96 Testamente von Frauen vor (45 in den AS, 10 in den AC, 38 in dem LT 772 und 3 in dem LT 773). Die Mehrheit der Testamente stammte von Männern.

${ }^{17}$ Vgl. Wysmulek, Jakub: Testament mieszczan krakowskich. Warszawa 2015. 
Bei jeder Eintragung kann man zwischen dem Kerntext (Haupttext) und einem oder mehreren Paratexten, also Texten, die sich vom Kerntext absetzen, unterscheiden. Auch der Haupttext kann graphisch in einen Großtext und einigen Kleintexten (die sich meist graphisch abzeichnen) unterteilt werden. ${ }^{18}$ Bei der Untersuchung der Sinnstruktur der Texte, der jeweiligen makro- und mikrostrukturellen Elemente, kann man auf die Konzeption von Teun van Dijk zurückgreifen. ${ }^{19}$ Bei der Analyse privater Testamentsurkunden beziehe ich mich auf die klassische Urkundenstruktur, die aus der Diplomatik bekannt ist. Außerdem ziehe ich die im Mittelalter populären Formularbücher heran. Anders muss man mit den zwei erstgenannten testamentarischen Texttypen umgehen, da diese dem bisherigen Schema nicht folgen. Der terminologischen Verständlichkeit halber greife ich aber auch in diesem Fall, wenn es möglich ist, auf die aus der klassischen Diplomatik bekannten Begrifflichkeiten zurück. Dabei wird in den Kerntexten der betreffenden Eintragungen nur die Dispositio vollständig realisiert, während das Protokoll, ein Großteil der Substantia (v.a. die Arenga) und teilweise auch das Eschatokoll (obwohl in der Abschrift meistens gekürzt) nur in den Testamentsurkunden ausgeführt werden. Im Folgenden wird der zentrale Teil eines jeden Testaments, also die Dispositio, nach ontologischen und pragmatischen Elementen befragt.

\subsection{Fakultative semantische Elemente in der Dispositio}

Nach dem Formularbuch Deutsche Rhetorica ${ }^{20}$ und im Einklang mit dem klassischen Urkundenschema fängt der Teil Dispositio

${ }^{18}$ Vgl. Greule, Albrecht/Reimann, Sandra: Basiswissen Textgrammatik. Tübingen 2015.

${ }^{19}$ Vgl, van Dijk, Teun Adrianus: Macrostructures. An interdiciplinary Study of Global Structures in Discourse, Interaction, and Cognition. Hillsdale/ 1980.

${ }^{20}$ Ohne Autor: Formulare und deutsch Rhetorica. Strassburg 1486. Vgl. auch Spáčilová, Libuse: Deutsche Testamente von Olmützer Bürgern. Wien 2000. 
nach der Narratio an. Er wird häufig durch ein Verweiselement eingeleitet und setzt sich aus einzelnen Verfügungen (hauptsächlich Vermächtnissen), der Pertinenzklausel (die sich auf die übrige Habe bezieht) und der Veränderungsklausel zusammen. Die tatsächliche Ausführung dieses zentralen Teils eines jeden Testaments umfasst aber noch weitere semantische Elemente, die erst auf der Ebene der Substruktur realisiert werden (können). $\mathrm{Zu}$ diesen sind sowohl ,freie ' als auch in einzelne Artikel bzw. in einzelne nicht vermögensrechtliche Verfügungen gebundene Sequenzen mitzurechnen. Unter einem, Artikel' verstehe ich einen Akt, der konkrete testamentarische Bestimmungen enthält. Dieser wurde in den vorliegenden Testamenten entweder in einem Satz bzw. in einem Teilsatz formuliert, oder erscheint als Teil einer längeren Satzperiode. Außer der Donation, die den Charakter dieser Einheit des testamentarischen Textes determiniert, können weitere semantische Elemente vorkommen, die sich zum Teil kategorisieren lassen. ${ }^{21}$ Ich liste im Folgenden einige auf:

I) Die Konditionalklauseln. Die größte Gruppe der Klauseln bilden Konditionalklauseln, die Bedingungen beinhalten, die vorkommen müssen, damit die im Artikel formulierte Bestimmung realisiert wird. $\mathrm{Zu}$ diesen gehören:

- Hochzeitsklauseln, zu denen alle Klauseln gehören, die sich auf eine Eheschließung beziehen, entweder in positiver oder in negativer Form (negative beziehen sich dann meistens auf den verbleibenden Ehepartner, zumeist die Frau, und können auch als Witwenklauseln realisiert werden). Vgl. hierzu: „Ab mich got czu syne(n) gnad(i)n neme, vnd mein weip yn erem wittwenstule blebe siczczin $[\ldots]$. “22

${ }^{21}$ Vgl. auch Bieberstedt, Andreas: Textstruktur - Textstrukturvariation - Textstrukturmuster. Lübecker mittelniederdeutsche Testamente des 14. und 15. Jahrhunderts. Wien 2007. Die angeführten Zitate entstammen früheren Belegen.

${ }^{22}$ Acta Scabinalia (= AS) III, 19.05.1393. 
- Kindesklauseln, die Rücksicht auf noch ungeborene Kinder nehmen bzw. die Erbfolge im Fall der Geburt des Nachwuchses (meistens des Testators) regeln (sie sind auch in negativer Form möglich). Vgl. hierzu: ,[...] alzo verre als ich ane vrochte vorscheide [...]."23

- Überlebensklauseln, die als Bedingung das (Noch-) Am-Leben-Sein der bedachten Personen bzw. ihrer Nachfolger formulieren. Vgl. hierzu: ,[...] is daz her mich obir lebit [...]. "24 Für das in Frage gestellte Überleben des Testators vgl.: ,vnd ap got ab(er) em gebote vf dem Romwege [...]." ${ }^{\circ 25}$

- Altersklauseln, die die Erbnahme von der Erreichung eines bestimmten Alters durch den Bedachten abhängig machen: Ende des 14. Jahrhunderts betrug dieses Alter meistens 24 Jahre. Vgl. hierzu: „Ab myn zon gewuchst vnd sein 1-teile des guttis welde habin dem sal man nichtis gebin [...], her kome den czu xxiiij Jaren [...]. ${ }^{\text {(26 }}$

- Führungsklauseln, die ein bestimmtes Verhalten, eine konkrete Lebensweise seitens des Bedachten als eine Bedingung der Erbnahme benennen. Meistens handelt es sich in den protokollartigen testamentarischen Eintragungen und Eintragungen mit der Abschrift einer Testamentsskizze darum, dass die Kinder der Frau und/ oder Vormunden folgen sollen (mit eventueller Sanktion im negativen Fall) oder darum, dass eine Liegenschaft nicht verkauft werden darf. In den eingelegten privaten Testamentsurkunden üblich sind dagegen auch ausgebaute Beschreibungen des erwünschten Verhaltens. Vgl. hierzu folgenden Beleg, in dem eine kirchliche

${ }^{23}$ AS III, 24.01.1393.

${ }^{24} \mathrm{Vgl}$. ebenda.

${ }^{25}$ AS, III 28.11.1393/31. 01.1393.

${ }^{26}$ AS, III 19.05.1393. Oft ohne die Angabe eines Alters (auch als Temporalsatz möglich). 
Stiftung (fundatio) samt einer genau bestimmten Vorgehensweise des Priester verfügt werden ${ }^{27}$ :

vnd noch zeine(m) tode zal der zelbig(e) czins gewant werden mit rothe der vormu(n)den vnd des Ersamen rot(is) czw krokaw czw dem dewtcz(e)n Prediger alhir yn der phar(e)r kyrche czw vnse(e)r liben fraw(e)n Alzo wer do rechter Prediger ist auff dÿ czeit der zal den zelbigen czinsz haben vnd eÿn allen predigt(e)n vor meine zele bitten dorczw all(e) wochen czwn messen lezen was vor messen her wil, vnd sülch(e)n czins zal her haben dy weile her lebet der vrsach(e)n halben zo ey(n) prediger aldt wörde ader yn krangheit qweme das her nicht predigen mócht das her hette wor von czw leben vnd zo der stürbe zo zal sủlch(e)n czins der hab(e)n der die dewtczer Prediger ist yn mossen als ebenAuch zal der zelbig(e) prediger der sủlchen czinsz alzo haben wörde nicht von hyne(n) czihen sunder bey der kyrchen bleiben bisz an zeyn ende, vnd zo her wezens wegk czòge vnd yn eine(n) halben ior(e) nicht wider qweme denn(e) baldt zal der prediger welch(e)r auff dy czeÿt Prediger wer(e) sulchen czins ÿn mossen als oben haben vnd besitczen $[\ldots]$.

- als eine Führungsklausel im weiten Sinne kann auch die Abfindungsklausel gelten, die das Recht an dem Erbe teilzunehmen, von der Abfindung des Bedachten mit dem ihm im Testament zuerkannten Erbe abhängig macht. Vgl. hierzu:

Vnde ist das meyne tachter dyse obgeschribene meyne beschedu(n)ge nicht anneme vnd hindern adir anfechtin wörde. so sal sy czu den obgenante(n) meyne(n) dreyteilen des obgena(n)ten hausis keyn recht habin. vnd sol do von awsgeslossen seyn czu ewige(n) tagen $[\ldots] .{ }^{28}$

- Berufsklauseln, die die Erbnahme mit der konkreten Berufswahl des Bedachten (bzw. der Standeswahl,

${ }^{27}$ AS IX, 19.05.1508.

${ }^{28}$ AS VI, 10.02.1441. 
wenn es sich um ein Priesteramt handelt) in ein Abhängigkeitsverhältnis setzen. Vgl. hierzu: ,Jt(em) Jch gebe Joh[(ani)s] Jorge Morre(n)steyns dyn(er) eyne(n) Pelcz a ap her prister wirt ${ }^{\star 22}$.

II) Weitere Klauseln. Weitere Klauseln, die in testamentarischen Artikeln vorkommen (können), haben keine konditionale Bedeutung, beziehen sich auch nicht mehr auf den Bedachten, sondern auf das Erbe selbst. Zu diesen können folgende gezählt werden:

- Todesfallanweisungen, die bestimmen, wie über die konkrete Erbmasse nach dem Ableben des Bedachten verfügt wird. Vgl. hierzu: ,[...] vnd ab vns(er) hirre myne(m) zon czu syne(n) gnod(i)n neme was da guttis bleibit das sullen dy vormu(n)de gebin xx m(a)rc(en) czur Mogil czum gewelbe des Crucegangis. " ${ }^{\text {30 }}$

- Folgeanweisungen, die die Erbfolge der konkreten Erbmasse für einen längeren Zeitraum bestimmen (auch wenn z.B. der Ehepartner lediglich das Nutzungsrecht zu Lebzeiten erhält und danach die Frage nach der Erbfolge erneut entstehen könnte). Vgl. hierzu: ,[...] vnd nach der fraw(i)n tode sull(i)n dy nochgeschreb(i)n vormu(n)der, das hus vorkawfen vnd sull(i)n das nochgeschreb(i)n gelt geb(i)n p(er)sone(m) vnd Steten den es beschreb(i)n stet [...]. “31

- Modifikationsanweisungen, die konkrete Anweisungen für die Legatenvollstreckung geben (auch wenn es sich um komplizierte Erbmassenverhältnisse handelt, Verfügung über Zinsen auf Immobilien mit Wiederkaufsrecht oder Anteile auf Erzgüter). Vgl. hierzu ${ }^{32}$ :

\footnotetext{
${ }^{29}$ AS IV, 25.09.1416.

${ }^{30}$ AS III, 19.05.1393.

${ }^{31}$ AS III, 08.01.1395.

${ }^{32}$ AS III, 22.08.1393.
} 
vnd $v m(m) e$ dy zelgerethe dy meyn h(er)re got habe zey(n) sele hat gemacht vnd gegebin czu de(m) altar xxv jar vnd iczlich jar sal man gebin alzo vil als man von der kamer czu czinse gibt durc[h] got vz der kamer vnd czu de[r] xxv jar gebe ich $\mathrm{x}$ jar vnd von den $\mathrm{x}$ jaren ge zal man alzo vit gebin also [v]il als man gibt von den egenen(ten) xxv Jaren vnd daseyn dank zeyn sal. $^{33}$

- Funktionsanweisungen, die konkrete Zwecke der bedachten Erbteile/der Erbmasse angeben. Vgl. hierzu: ,[...] von dem Czinse sal ma(n) alle Jar vier tuch sneiden arme(n) leuthe(n) ie dy q(ua)temp(er) eyn tuch, vnd eyne $\mathrm{m}(\mathrm{a}) \mathrm{rg}$ arme leuthe czuspeisen vnd dis sal ewig bleiben." ${ }^{634}$

- Darüber hinaus kann man Elemente der Artikel isolieren, die aus der Sorge um das Seelenheil des Testators resultieren. Es sind allgemein formulierte Heilsformen . Vgl. hierzu: ,[...] czwu m(a)rg alle ior wenden ad(er) an dy heyligi(n) werk der barmherczikeyt czu troste meyner zele vnd meynis vorderigin wirtis ${ }^{\text {‘35 }}$. Zu den an die Bedachten gerichteten Fürbitteanweisungen vgl.: ,[...] czu notdorft der Bruder desselb(e)n Closters, daz dy ouch mich in ewigem gedechtnis hab(e)n, von got vor mich beten." ${ }^{\text {(36 }}$

- Als letzte in der Donatio sich wiederholende fakultative Klauseln sind die Überschuss- und Defizitklauseln zu nennen, die denjenigen Fall regeln, wenn die zu verfügende Habe sich als zu hoch bzw. zu niedrig erweist. Vgl. hierzu: „,[...] vnd ab icht do worde sein von farndem gutte obir di erbe $[\ldots] .{ }^{6}{ }^{637}$

${ }^{33}$ Durchkreuzungen, eine Variation der grammatischen Person und weitere Verschreibungen. Dabei handelt es sich um eine Czedelabschrift. ${ }^{34}$ AS III, 28.11.1393/31.01.1393.

${ }^{35}$ AS III, 24.01.1393.

${ }^{36}$ AS VI, 10.06.1440.

${ }^{37}$ AS III, 19.05.1393. 
Zahlenmäßig wurden bei der Analyse alle Texttypen der testamentarischen Eintragungen bis 1550 aus den Gerichtsbüchern (AS) erfasst, wobei nach dem Vorkommen der einzelnen Klauseln gefragt wurde. Die Ergebnisse der Analyse sind in der Tabelle erfasst. Insgesamt sind 177 AS-Testamente untersucht worden, davon: 58 Czedelabschriften, 83 protokollartige Eintragungen, 33 eingetragene private Bürgerurkunden, 3 Dokumente der Behörde:

\begin{tabular}{|c|c|c|c|c|c|c|c|}
\hline \begin{tabular}{|l|}
$\begin{array}{l}\text { konditionale } \\
\text { Klauseln }\end{array}$ \\
\end{tabular} & $\begin{array}{c}\text { Czedel- } \\
\text { abschrift }\end{array}$ & Protokolle & $\begin{array}{l}\text { eingelegte } \\
\text { Testam. }\end{array}$ & $\begin{array}{c}\text { weitere } \\
\text { Klauseln }\end{array}$ & $\begin{array}{l}\text { Czedel- } \\
\text { abschrift }\end{array}$ & Protokolle & $\begin{array}{l}\text { eingelegte } \\
\text { Testam. }\end{array}$ \\
\hline \begin{tabular}{|l|} 
Hochzeits- \\
klausel
\end{tabular} & 6 St. $/ 10 \%$ & 7 St./8\% & 14 St./42\% & $\begin{array}{l}\text { Todesfall- } \\
\text { klausel }\end{array}$ & 14 St. $/ 24 \%$ & 12 St. $/ 14 \%$ & 12 St./33\% \\
\hline Kindesklausel & 8 St./14\% & $3 \mathrm{St} . / 4 \%$ & 3 St./9\% & $\begin{array}{c}\text { Folge- } \\
\text { anweisung }\end{array}$ & 7 St. $/ 12 \%$ & 7 St../8\% & 9 St. $127 \%$ \\
\hline \begin{tabular}{|l} 
Überlebens- \\
klausel \\
\end{tabular} & 14 St./24\% & 18 St. $/ 22 \%$ & 3 St. $/ 18 \%$ & $\begin{array}{c}\text { Modifikations } \\
\text {-anweisung } \\
\end{array}$ & 18 St. $/ 31 \%$ & 34 St. $/ 41 \%$ & 14 St. $/ 42 \%$ \\
\hline Altersklausel & 6 St. $/ 10 \%$ & 10 St. $/ 12 \%$ & 6 St. $/ 18 \%$ & $\begin{array}{c}\text { Funktions- } \\
\text { anweisungen }\end{array}$ & 25 St. $/ 43 \%$ & 28 St. $/ 34 \%$ & 19 St. $/ 58 \%$ \\
\hline \begin{tabular}{|l|} 
Führungs- \\
klausel
\end{tabular} & 8 St. $/ 14 \%$ & 5 St. $/ 6 \%$ & 10 St. $/ 30 \%$ & Heilsformel & 6 St. $/ 10 \%$ & 23 St. $/ 28 \%$ & 10 St. $/ 30 \%$ \\
\hline $\begin{array}{l}\text { Abfindungs- } \\
\text { klausel }\end{array}$ & 5 St. $17 \%$ & 12 St. $/ 14 \%$ & 10 St. $/ 30 \%$ & Fürbittklausel & 4 St. $/ 7 \%$ & 9 St. $/ 11 \%$ & 11 St. $/ 33 \%$ \\
\hline Berufsklausel & 0 & $2 \mathrm{St} . / 2 \%$ & 5 St. $/ 15 \%$ & $\begin{array}{c}\text { Überschuss- } \\
\text { und Defizit- } \\
\text { klausel }\end{array}$ & 8 St. $/ 14 \%$ & 6 St. $/ 7 \%$ & 4 St. $/ 12 \%$ \\
\hline
\end{tabular}

Tab. 1: Klauseltypologie in testamentarischen Texten aus den Krakauer Gerichtsbüchern (bis 1550)

Die in allen Texttypen der Testamente häufigsten Klauseln sind die Funktionsanweisungen, die Modifikationsanweisungen und die Überlebensklauseln. Einige Klauseln werden öfter in den Texttypen, die von privaten Schreibern stammen (besonders in den privaten Testamentsurkunden) eingebunden, als in den protokollartigen Eintragungen der Kanzleimitarbeiter. Es handelt sich v.a. um die Hochzeitsklausel, die Führungsklausel, die Abfindungsklausel, die Folgeanweisungen und die Fürbittklausel. Diese Unterschiede können daher rühren, dass die sie anfertigenden bezahlten Schreiber darauf bedacht waren, alle Elemente, die der jeweilige Testator ihnen diktierte, getreu in den Text aufzunehmen. Deswegen überraschen auch die längeren 
Partikular-Narrationen einiger Artikel bzw. nichtvermögensrechtlicher Bestimmungen kaum. Die Zeit der Entstehung des Testaments und das Geschlecht des Testators haben dagegen keinen sonderlichen Einfluss auf das Vorkommen der fakultativen Elemente.

III) Zusätzliche assertive und direktive Akte. Unter den drei Texttypenzeigensich gravierendeUnterschiedeimdurchschnittlichen Umfang der Texte. Verglichen werden können nur die zwei, die parallel in derselben Zeit üblich waren, d.h. Protokolle und eingelegten Bürgerurkunden in der ersten Hälfte des 16. Jahrhunderts (die Czedel sind zu dieser Zeit bereits fast aus dem Gebrauch gekommen): Während der Durchschnittsumfang der Protokolle (anhand der von mir durchgeführten Zusammenstellungen und Transliterationen) ca. 500 Computerzeichen beträgt, belaufen sie sich bei den Männertestamenten auf 1.400 bzw. bei den Frauentestamenten auf 1.280. Die Unterschiede im Umfang sind allerdings nicht nur durch die Vorkommenshäufigkeit bestimmter Klauseln bedingt. Testamente müssen also noch weitere Elemente beinhalten, die auf diesen Sachverhalt Einfluss nehmen: Zusätzliche assertive und direktive Akte sind in dem Teil Dispositio der Krakauer Testamente sowohl als ,freie Substrukturelemente als auch als Teile der Artikel zu finden. Im letzten Fall weise ich auf die in einigen Testamenten vorkommende Partikular-Narratio der einzelnen Artikel hin, welche die Umstände der Donation benennen bzw. die Erbteile näher beschreiben. Üblich war vor allem die Nennung des Grundes für die jeweilige Donation (meist eine größere als eine übliche in ähnlichen Verhältnissen). In den Gerichtsbüchern (AS) konnten Begründungen für einzelne Donationen in 3 Czedeln $(5 \%)$, in 11 Protokollen (13\%) und in 12 eingelegten Testamentsurkunden (36\%) festgestellt werden. Es sind 
meistens Danksagungen für treue Dienste bzw. für das Mitwirken im geführten Handelsinteresse oder aber für die treue Mitwirtschaft des Ehepartners. Möglich sind auch Formulierungen, die im Übergangsbereich zur Benennung eines Grundes, einer Funktionsanweisung und einem indirekten direktiven (bzw. perlokutionären) Akt angesiedelt sind:

Jtem Vlrich hozen meyne(m) SchwogeR zal man aws richtenSechs hundertguld(en) czw halb(e)nschoken sulch gelt geb ich ym vor das das ich ym schuldigk byn eyn teil vnd das ander aws fruntschafft do mit her deste grosse(e)rn fleisz ankeren wirt yn meyne(n) geschefften, noch meyne(m) tode neb(e)n meynes bruders kyndern $\left[\ldots . .{ }^{38}\right.$

Vereinzelt finden sich - und das auch nur in den privaten Testamentsurkunden - direktive Akte, die nicht als Klauseln zu Artikeln zu verstehen sind:

Vnnd weytt(e)rgebyth ich vnnd bith meyne(n) son Jorge d(as) er sal dy kauffkamer wol besehe(n) das dy kauffkamer(n) nicht wüste stee Vnnd so er sach wer das der eldsteson Jorg ey(n) weib neme(n) wolt das dy kauffkamer(n) nicht wuste stee. So sal er czw ym neme(n) den and(e)rn son Stenczel sey(n) bruder vnndt ym vnd(er)wesey(n) vnd lerne(n) das er sich yn dem handel eyst zW richte(n). das den kind(e)rn ally(n) nicht an gutt(e)rn abgingk $[\ldots] .{ }^{39}$

Außer den Artikeln sind in den Krakauer Testamenten auch nichtvermögensrechtliche Bestimmungen zu finden: Diese haben meistens den Ort des Begräbnisses, eventuell mit weiteren detaillierten Anweisungen zu dessen Ausführung zum Gegenstand. Zu den , freien“ Elementen rechne ich auch solche, die in keinem direkten

${ }^{38}$ AS IX, 14.06.1507.

${ }^{39}$ AS X, 23.02.1521. 
Zusammenhang mit den im Testament benannten Donationsakten stehen, also keinen Teil eines Artikels ausmachen. Hierzu zählen Bekenntnisse, Informationen über frühere Vergabungen bzw. Ausrichtungen der bereits mündigen Kinder wie auch integrierte längere Verzeichnisse der Schulden oder Verzeichnisse der Habe. Adaptative Textmuster bürgern sich im Laufe des 15. Jahrhunderts ein. Über den Charakter der Rechtshandlung und dieWahl der Textsorte für die Verschriftlichung entscheidet dann immer der Donationsakt als der Dominanzakt eines komplexen Textmusters. Es fällt jedoch auf, dass die Verzeichnisse der Habe bzw. der Schulden sich nur selten als integrierte Teile der privaten Testamentsurkunden finden, die in diesem Texttypus der Testamente durch Hinweise auf externe Unterlagen kompensiert werden (in den AS: Aufzählung der Habe in den Czedel: 0-mal, Aufzählung der Schulden in den Czedel: 2-mal, Aufzählung der Habe in den Protokollen: 2-mal, Aufzählung der Schulden in den Protokollen: 13-mal, Aufzählung der Habe in den privaten Testamentsurkunden: 3-mal, Aufzählung der Schulden in den privaten Testamentsurkunden: 3-mal).

\section{Ziel des Testierens}

Testamente werden immer dann verfasst, wenn man die durch das gültige Recht geregelte Erbfolge modifizieren will. Dank diesem Rechtsinstrument konnte primär die Anwartschaft der durch das Gesetz vorgesehenen (,natürlichen') Erben vermehrt bzw. verkürzt werden und ggf. Erbnehmer ernannt werden, die außerhalb dieses Kreises stammten. In der mittelalterlichen rechtlich-sozialen Realität war in diesem Zusammenhang besonders die Situation der Frauen vom Wohlwollen ihres Ehemannes 
abhängig, da sie am normalen Erbgang üblicherweise nicht teilnahm. Als Absicherung diente ihnen lediglich die bei der Eheschließung beschlossene Morgengabe und das eventuell angefertigte Testament. Dank diesem Instrument konnte also die Verteilung der hinterlassenen Habe neu geregelt werden, und zwar anhand individueller Entscheidungen. Diese richteten sich nicht mehr nach dem Interesse der Stammfamilie, sondern berücksichtigten zunehmend die tatsächlichen Beziehungen der Individuen untereinander, also diejenigen Menschen, dem der Testator/die Testatorin besonders nahestanden oder denen er/sie die Vermehrung seines/ihres Wohlstandes verdankte. In etlichen Fällen der eingebrachten letztwilligen Bürgerurkunden wurden explizite assertive Äußerungen mit Begründung der Abweichung von der üblichen Erbfolge angeführt. Jegliche Modifikation des üblichen Erbgangs bedeutete nämlich zwangsläufig, dass wenigstens ein Teil der gesetzlichen Erbnehmer (oder auch alle) benachteiligt wurden z.B. eine vermehrte Donation zugunsten der Frau (d.h. über die ihr zustehende Morgengabe). Vgl. hierzu:

[...] wmb(e) der trew wille dy sy bey ÿm yn seyner krankeyt gethan hattoder zugunsten des Sohnes, der zur Wohlstandvermehrung der Familie beigetragen hat: LT772 17.06.1479: Zum ist(e)nn hat h(e)r bescheid(e)n h(er)n Jorg(e)n seine(m) elst(e)n zone von farind(e)n gutt(i)rn Tausint guld(e)n dorum(m)e das h(e)r dy bey im sain ey(n) din(e)r getrewlich direrbit hat mit sein(e)r gross(e)r muhe vn(d) arbeit $[\ldots] .^{40}$

Besonders wenn es sich um das sog. wohlgewonne Gut handelte, stand dem Testator frei, über seine Habe postmortalisch zu verfügen. Falls es sich aber um die sog. Stammerben handelte, wurde den Testierern seitens der Stadtbehörde besonders scharf auf die Finger geschaut. Siehe dazu die Vorgehensweise der Räte etwa im Text LT 772 vom 14.04.1458 protokolliert: ,[...] do wart her gefrogit czum irsten czum and(er)n vnd czum

${ }^{40}$ AS IX, 01.04.1512. 
dirtten mole ap dy obgeschrebene ordenu(n)ge sein freyer guttir wille were her solde gancz in kleynem stucke gedrungen vnd getwungen seyn".Ziel der Verfügungen war meistens, jeglicheUnsicherheitenund Spannungen(keineZwietrachtzulassen) zwischen den Erbnehmern (besonders innerhalb der Familie) zu vermeiden. Ähnliche Phrasen kommen in 21 aller Krakauer Testamente vor.

Als ein sekundäres Ziel des Testierens kann die Sicherung der Erinnerung des Testators erachtet werden. Unter diesem Aspekt kann zum Teil auch die Sorge um das Seelenheil subsumiert werden, da in einigen Fällen hinter dem Gedanken des Gedächtnisses das Prinzip des do ut des(auf Deutsch: Ich gebe, damit du gebest) wirksam war. Vgl.: ,[...] das bescheide ich vnd befele is Margarethe meyner hausfraw(e)n. in deme getrewen das ich czu irre trow habe. daz sy meynir zele dovon getrewlich gut tuen sal ${ }^{“ 41}$ und „Jt(e)m alle Schoff dy ich ym forwergk habe Jungk vnd aldt sullen ir awsgegeben werden czw eyne $(\mathrm{m})$ gedechtnisse meyner mich nicht czW vorgessen. ${ }^{642}$ Es kann auch angenommen werden, dass die Idee, viele Erbnehmer zum Erbgang zu berufen, eben diesem Ziel gerecht werden sollte.

Eine tragende institutionelle Rolle bei der Sorge um das Seelenheil haben Klöster, Kirchen und sog. Werke der Barmherzigkeit. Das Veranstalten und Abhalten von Seelenmessen, aber auch Donationen zugunsten klerikaler Bauten und ihrer Insassen sollten - ähnlich dem Ablasshandel - Gott dazu bewegen, die arme Seele in das Himmelreich aufzunehmen bzw. das Leid im Fegefeuer zu verkürzen. Eine ähnliche Funktion hatten weitere Bestimmungen ad pias causa (zu frommen Zwecken). Reiche Beschreibungen, wie sie im Modus konkreter Zweckeinnerhalb einer Donationen vorliegen, finden sich in einigen privaten letztwilligen Bürgerurkunden:

\footnotetext{
${ }^{41}$ AS VI, 09.09.1440.

${ }^{42}$ AS IX, 14.06.1507.
} 
Item Mer sollen sye meyner selenn czw außgebenn vnnd ynn dye wergk der barmherczikayt wenndenn 100 floren dar vonn armenn lewtenn rogk hembder kauffen auch schu(e)ller dye studirenn wollen bu(e)cher czaigenn vnnd fu(e)nft armenn gebrechlichenn lewtenn stewer gebenn wye dye Exequutores sampt meyner hausfraw vors beste erkennen werdenn. ${ }^{43}$

Die Idee ist stets dieselbe: durch die Fürsprache der Armen soll ewiges Erbarmen erlangt werden. Zur großen Gruppe von Donationen für die Werke der Barmherzigkeit gehörten aber auch sog. Donationen für stege und wege, also für das gemeinschaftliche Wohl (in 14 Testamenten in den AS). Besonders Personen, die im Kreis der Stadtbehörden verkehrten, waren darauf bedacht, auch nach ihrem Tod an die Stadt zu denken und für ihre Bewohner zu sorgen - natürlich wieder nach dem Prinzip do ut des (in 179 Testamenten in den AS, plus 6 eventuellen nach einer Sterbeklausel; darunter 119 kombiniert mit Donationen zugunsten der Kirchen bzw. deren Vertretern, in 60 Texten nur Donationen zugunsten der Kirchen).

\section{Emotionalität in den Krakauer Testamenten: Spannungen und Harmonie in der Familie}

Bereits anhand der postmortalen Verteilung der Güter kann man einige Schlussfolgerungen in Bezug auf die Bevorzugung einiger Familienmitglieder ziehen. Diese entsprechen aber nicht in jedem Fall der Wirklichkeit. Um nämlich ein ,echtes“ Situationsabbild rekonstruieren zu können, müsste man alle Donationen des jeweiligen Testators zugunsten der jeweiligen Person während seines Lebens berücksichtigen, nicht nur die letztwilligen Bestimmungen. Da aber die wissenschaftliche Erhebung solcher Daten so gut wie unmöglich ist und daher nicht vorliegt (und häufig auch nicht anhand der Stadtbücher nachvollziehbar ist), ist diese Schlussfolgerung äußerst vage. In privaten Bürgerurkunden kann man dagegen nicht selten auch Angaben zu

${ }^{43}$ Darunter in LT 772 z.B. 19.03.1540, Datierung unsicher, S. 276. 
diesen früheren Handlungen finden, die auch einige Modifikationen der normalen Erbfolge erklären (vgl. die Ausrichtung besonders der Töchter - als bei der Heirat anerkannte Teil der Habe, die vom Erbteil abgerechnet werden kann).

In einigen Testamenten finden sich aber gut ausgebaute Begründungen seitens des Testators, um seine Einstellung einigen Familienmitgliedern zu erläutern. Diese werden besonders dann sichtbar, wenn die Interessen der Familie durch eingeheiratete Mitglieder gefährdet werden können. Als Beispiel kann man hier das Testament AS XII, 14.04.1540 betrachten, in dem sich der Hinweis findet, dass die Tochter des Testators mit „eynem mann Jacob guldennsternn der ynn seyner narung gar abgekommen ist" verheiratet ist und deswegen nichts verkaufen und entwenden darf, solange dieser lebt.

Die Angaben zu den Spannungen innerhalb der Familie finden sich nicht nur in privaten Bürgerurkunden (in 5 Testamenten in den AS), sondern können auch in den vorgelegten Czedeln (Testamentsvorlagen) angetroffen werden (in 2 Testamenten in den AS). Vgl. hierzu folgenden Auszug aus dem Testament:

Von alle den obgenanten gutern slisse ich aus meyne tachter dorothea vnd Stanislalben iren man. daz sy dorczu keyn recht haben sullen wenn sy vmb mich keyn gutis vordynet haben vnd leydigen mich tegelich mit worten vnd mit werken. vnd hindern mich daz ich meyn testament nicht machen sulde. czu zelikeit meyner zele. ${ }^{44}$

Auch in protokollartigen Testamenten finden sich Äußerungen, die Spannungen in der Familie preisgeben (in 3 Testamenten in den AS). Vgl. AS X, 22.08.1513, in dem die Rede davon ist, dass der Mann in der Hauswirtschaft nicht geholfen hat und auch nicht wollte, dass die Frau testiert.

Testamente, und zwar hauptsächlich private Bürgerurkunden, verweisen nicht nur auf Konflikte, sondern lassen auch harmonische Verhältnisse in einer Familie erkennen. Einen

${ }^{44}$ AS VI, 27.05.1440. 
zentralen Platz nehmen die Gefühle zwischen Eheleuten ein, die in den eingebrachten Testamentstexten bzw. Vorlagen zum Tragen kommen. Die Genauigkeit der Wiedergabe der in einer privaten Testamentsurkunde gefassten Worte erlaubt auch die Verschriftlichung einiger emotional gefärbten Töne (in den AS insgesamt in 17 Texten: in 13 privaten Testamentsurkunden, in 2 Czedeln und 2 Protokollen). Diese manifestieren sich auf sprachlicher Ebene vornehmlich durch die verwendeten Attribute (etwa das Adjektiv lieb in 13 Texten in den AS; darunter auch lieb in Bezug auf Gebrüder und Schwäger) oder durch eine Beschreibung der Eigenschaften (vor allem die Treue und die Hingabe) sowie des Handelns der Ehepartner. Befremdend wirken hingegen längere emotionale Passagen in den Protokollen. Vgl.: „Ansechend auch trew lib wnd gunst ires liben mannes zo getrauet szie im auch ire sel [...].“45

\section{Die kulturelle und gesellschaftliche Sensibilität der Testierer}

Im Rahmen des vorliegenden Beitrags kann nur kurz auf einen wichtigen Aspekt hingewiesen werden, in dessen Rahmen die Krakauer Testamente einige neue Einsichten bieten. So lassen sich vor allem die Anweisungen zur Erziehung und (Aus-) Bildung der Kinder des Testators (in 8 Texten in den AS, und zwar in 5 privaten Testamentsurkunden und 3 Protokollen) und die Donationen zugunsten verschiedener gesellschaftlicher Einrichtungen wie Zechen und sozial benachteiligten Schichten (in Texten in den AS, und zwar in 2 privaten Testamentsurkunden und 4 Protokollen) als Indizien der Mentalität der einzelnen Testierer auslegen. Vgl. hierzu: „Vnder arme antwerglewte des hantwergs der Zatler desir Stat vmb gottis wille“. ${ }^{46}$ Die Anweisungen in Bezug auf die Bildung (Studium, Unterstützung der Ausbildung) finden sich interessanterweise im ganzen Textkorpus hauptsächlich erst im 16. Jahrhundert.

${ }^{45}$ Ebenda.
${ }^{46}$ AS VI, 01.12.1458. 


\section{Schlussbemerkungen}

Obwohl Testamente einen formalen und damit rein sachlichen Charakter haben, verweisen sie zwischen den Zeilen auch auf Privates. ${ }^{47}$ Die variablen sozialen Beziehungen und Verhältnisse (die soziale Stellung, die Bildung, das unterschiedliche Ausmaß des Reichtums usw.) haben nach dem Sprachhistoriker Józef Wiktorowicz zwar keinen direkten Einfluss auf die sprachliche Ausgestaltung der konventionalisierten Form der Textsorte ,Testament' im mittelalterlichen Krakau, sie bedingen jedoch eine gewisse Variabilität der einzelnen Textexemplare und haben Einfluss auf das Vorkommen fakultativer Elemente in den testamentarischen Texten.

Meine These ist: In einer Epoche, in der sich die Menschen im Zentrum Europas von den aufgezwängten Familienverpflichtungen befreien, sind sich die Testierer gleichzeitig ihres Einflusses auf die Güterteilung nach ihrem Tod zunehmend bewusster und stellen häufiger Wünsche und Bedingungen an die Bedachten (vgl. die anwachsende Häufigkeit der Heirats- oder der Führungsklauseln). Über die Rechtskraft dieser Elemente kann ich als Sprachhistorikerin jedoch nicht entscheiden, denn diese Frage ist primär an Rechtshistoriker adressiert.

Die erwähnte Variabilität der Textexemplare realisiert sich auch in der unterschiedlichen Formulierung der sich wiederholenden Klauseln. Diese Beobachtung betrifft aber nicht nur private Testamentsurkunden, sondern auch die von professionellen Kanzleimitarbeitern verfassten protokollartigen testamentarischen Eintragungen, was mit den heutigen Vorstellungen zu formelhaften Textsorten nicht übereinstimmt.

${ }^{47}$ Vgl. Kaleta-Wojtasik, Sławomira: Formelles und Persönliches in deutschsprachigen Testamenten der Krakauer Bürger aus dem 15./16. Jahrhundert. In: Hünecke, Rainer/Sandra Aehnelt (Hgg.): Kanzlei und Sprachkultur. Wien 2016, S. 49-62. 
Meine Untersuchung zur historischen Textsorte Testament zeigt jedoch, dass auch obligatorische Elemente, die zum Handlungs- bzw. Formulierungsmuster gehören, in mittelalterlichen Testamenten der Krakauer Bürger stark variieren. ${ }^{48}$ Dies ist mit den sprachlichen Gepflogenheiten der mittelalterlichen Kanzleien (insbesondere innerhalb der privaten Gerichtsbarkeit) vereinbar. Auch einige in den mittelalterlichen Testamenten zur Sprache kommenden Fragen wie die Sorge und das Seelenheil heben diese Testamente von den modernen Vertretern dieser Textsorte ab.

Mittelalterliche Testamente erscheinen teilweise als Dokumente privaten Charakters, als sog. Selbstzeugnisse. ${ }^{49}$ Diese Bezeichnung bezieht sich auf historische Quellen, die Informationen beinhalten bzw. Rückschlüsse über das private Leben ihrer Autoren, ihre Gefühle, Ansichten usw. erlauben. Der Historiker Eamon Duffy warnt jedoch davor, die Individualität dieser Textsorte zu überschätzen, da diese oft von professionellen Schreibern verfasst wurden, die auf eine persönliche Note bewusst verzichteten bzw. in ihrer Aussagekraft entschärften. ${ }^{50}$ Auf die Unterschiede zwischen den Texttypen der Testamente in Abhängigkeit von dem Ausführer habe ich bereits hingewiesen.

Meine bisherigen Untersuchungsergebnisse legen daher weitere prosopografische Ansätze bei der Analyse von Testamentstexten nahe. Die Prosopografie gehört nämlich auch zu den ontologisch-pragmatischen Aspekten, die im Folgenden kurz erwähnt werden sollen: Die von Jakub Wysmulek und von mir in meiner Dissertation gebotenen Zusammenstellungen

${ }^{48}$ Vgl. Smereka, Joanna: Textlinguistische Untersuchungen zu Testamenten der Krakauer Bürger vom 14. bis zur Hälfte des 16. Jahrhunderts (i. Dr.).

${ }^{49} \mathrm{Vgl}$. Schulze, Winfried: Ego-Dokumente. Annäherung an den Menschen in der Geschichte. Berlin 1996.

${ }^{50}$ Vgl. Duffy, Eamon: The Stripping of the Altars. Traditional Religion in England 1400-1580. New Haven 2005. Ein Hinweis darauf findet sich bereits bei Jakub Wysmułek, siehe Fußnote 17. 
Krakauer Bürgertestamente sind bei einem solchen Unterfangen insofern hilfreich, da sie wesentliche Informationen über die soziale Stelle des jeweiligen Testators, seinen Beruf sowie selbstverständlich auch über seine Familienmitglieder und seinen Besitz liefern. In einer mittelalterlichen Stadt wie Krakau, in der die Bürgerhierarchie von grundlegender Bedeutung war und ungefähr 10 Prozent der Stadtbevölkerung zur Schicht der sog. Patrizier gehörte, lassen sich Informationen über die soziale Stellung der einzelnen Testamentsautoren und -autorinnen auch anhand des sozialen Beziehungsnetzes herauspräpapieren. Genügend Indizien hierfür finden sich direkt im Text selbst oder auch indirekt: über die Namen und sie soziale Stellung der ernannten Vormunde bzw. Exekutoren und der Topografie der Liegenschaften, da die Häuser am Ring und in dessen Umgebung eigentlich nur in der Hand der Patrizier sein konnten.

\section{Literatur}

\section{Primärliteratur}

Formulare und deutsch Rhetorica. Strassburg 1486.

Textbücher Krakauer Testamente

\section{Sekundärliteratur}

Bieberstedt, Andreas: Textstruktur - Textstrukturvariation Textstrukturmuster. Lübecker mittelniederdeutsche Testamente des 14. und 15. Jahrhunderts. Wien 2007.

Duffy, Eamon: The Stripping of the Altars. Traditional Religion in England 1400-1580. New Haven 2005.

Dunin-Dudkowska, Anna: Testament jako zwierciadto kultur. Lublin 2014.

Dijk van, Teun Adrianus: Macrostructures. An interdiciplinary Study of Global Structures in Discourse, Interaction, and Cognition. Hillsdale/1980. 
Greule, Albrecht/Reimann, Sandra: Basiswissen Textgrammatik. Tübingen 2015.

Heidegger, Martin: Sein und Zeit. Tübingen 1927.

Kaczmarczyk, Kazimierz (Hg.): Księgi przyjęć do prawa miejskiego 1392-1506. Kraków 1913.

Kaleta-Wojtasik, Sławomira: Formelles und Persönliches in deutschsprachigen Testamenten der Krakauer Bürger aus dem 15./16 Jahrhundert. In: Hünecke, Rainer/Sandra Aehnelt (Hgg.): Kanzlei und Sprachkultur. Wien 2016, S. 49-62.

Krause, Wolf-Dieter: Text, Textsorte, Textvergleich. In: Adamzik, Kirsten: Textsorten. Reflexionen und Analysen. Tübingen 2010, S. 45-76.

Meier, Jörg: Städtische Kommunikation in der Frühen Neuzeit. Historische Soziopragmatik und Historische Textlinguistik. Frankfurt am Main 2004.

Ostaszewska, Danuta/Cudak, Romuald (Hgg.): Polska genologia lingwistyczna. Warszawa 2008.

Sandig, Barbara: Stilistik. Sprachpragmatische Grundlagen der Stilbeschreibung. Berlin 1978.

Schulze, Winfried: Ego-Dokumente. Annäherung an den Menschen in der Geschichte. Berlin 1996.

Smereka, Joanna: Textlinguistische Untersuchungen zu Testamenten der Krakauer Bürger vom 14. bis zur Hälfte des 16. Jahrhunderts (i. Dr.).

Spáčilová, Libuse: Deutsche Testamente von Olmützer Bürgern. Wien2000.

Stanislaw (Hg.): Systematyzacja pojęć w stylistyce. Opole 1992, S. 141-147.

Wysmulek, Jakub: Testament mieszczan krakowskich. Warszawa 2015.

Wiktorowicz, Józef: Kanzleistil als Eigenschaft sprachlicher Handlungsmuster. Sprachliche Handlungsmuster in kanzleisprachlichen Texten. In: Hünecke, Rainer/Sandra Aehnelt (Hgg.): Kanzlei und Sprachkultur. Wien 2016, S. 97-106. 
Wiktorowicz, Józef: Die Textsorte Testament in der deutschen Kanzleisprache in Krakau. In: Simmler, Franz (Hg.): Textsortentypologien und Textallianzen von der Mitte des 15. bis zur Mitte des 16. Jahrhunderts. Berlin 2004, S. 167-176.

Wojtak, Maria: Genologia testów użytkowych. In: Ostaszewska, Danuta/Romuald Cudak (Hgg.): Polska genologia lingwistyczna. Warszawa 2008, S. 339-352.

Wojtak, Maria: Gatunki prasowe. Lublin 2004.

Wojtak, Maria: O stylowym wariancie języka na przykładzie tekstów urzędowych. In: Gajda, Stanislaw (Hg.): Systematyzacja pojęć w stylistyce. Opole 1992, S. 141-147.

Ziegler, Arne: Städtische Kommunikationspraxis im Spätmittelalter. Historische Soziopragmatik und Historische Textlinguistik. Berlin 2003. 